\title{
A Sociolinguistic Analysis of the Use of Arabizi in Social Media Among Saudi Arabians
}

\author{
Ashwaq Alsulami ${ }^{1}$ \\ ${ }^{1}$ School of Languages, Literatures, and Linguistics, Bangor University, Wales, United Kingdom \\ Correspondence: Ashwaq Alsulami, School of Languages, Literatures, and Linguistics, Bangor University, Wales, \\ United Kingdom. E-mail: ashwaq.rj@hotmail.com
}

Received: August 27, 2019 Accepted: September 20, 2019 Online Published: October 28, 2019

doi:10.5539/ijel.v9n6p257 URL: https://doi.org/10.5539/ijel.v9n6p257

\begin{abstract}
The aim of this sociolinguistically-oriented study is to explore the Arabizi phenomenon which is characterized by spelling Arabic words using the Latin script. It is prevalent in the text-based computer-mediated communications among Saudi Arabians. The study focuses on why Arabizi is used, how, particularly in respect to with whom and in which topics, it is used, the attitudes of its users toward its use and the perceived advantages and disadvantages of its use. Using an online survey, data were collected from 241 participants, 72 of which were users of Arabizi. The findings revealed that the primary reasons for using Arabizi were its being a communication code among youths and a compensation for the lack of Arabic keyboard from technological devices as well as being more expressive than Arabic language. It was also found that Arabizi was primarily used to communicate with friends and individuals of the same age, but not with parents and older people or in formal relationships. In addition, the study revealed that Arabizi is used in occasional conversations and social matters, but not in academic, scientific, business, economic, religious or poetry and literature- related topics. Arabizi users were found to hold both positive and negative attitudes based on different advantages and disadvantages of the phenomenon. These findings will be discussed and recommendations for future research will be given.
\end{abstract}

Keywords: sociolinguistics, Arabizi, social media, Saudi youth

\section{Introduction}

Text-based computer and mobile phone mediated communications are very popular worldwide (Al-Salman, 2017). The nature of these communications and specifically how they impact language and give rise to different linguistic phenomenon has become the focus of a great body of research (Herring, 2012). This line of research is concerned with studying the characteristics of Computer-Mediated Communication (CMC) language and how it differs from the speaking and writing modalities of linguistic output (Crystal, 2005). Such research has revealed deviations (termed as textisms) from the standard form of a language such as English (Herring, 2012). Textisms that have been observed included, for example, non-standard typography such as the use of special non-alphabetic symbols and non-standard orthography such as the use of non-standard spelling based on the phonetic pronunciation of words (Crystal, 2008; Farina \& Lyddy, 2011; Thurlow \& Poff, 2011). This line of research also tried to explore the sociolinguistic aspects of these textisms to understand the causes and factors leading to their rise in CMC language. The proposed causes included to overcome technical limitations such as the message size, to sound informal in order to ensure intimacy or to construct social identity and perform group membership (e.g., Androutsopoulos, 2011; Tagg, 2009; Thurlow \& Poff, 2011). Textisms have been observed in the CMC communications cross-linguistically. For example, they have been observed in text-based communications among youths in Germany (e.g., Bieswanger, 2008), Netherlands (e.g., Verheijen, 2017), France (e.g., Lanchantin, Simoës-Perlant \& Largy, 2014), Slovenia, Croatia and Serbia (e.g., Miličević, Ljubešić, \& Fišer, 2017). In some countries, whose national language uses a non-Latin script in its writing system such as Greek and Arabic, one of the observed textisms is spelling out words by the Latin script (e.g., Bies et al., 2014; Spilioti, 2009). It is the language arising from such textism in Arabic, which is termed Arabizi, and particularly its sociolinguistic aspects that are the focus of the present study.

\section{Research Objectives}

The aim of this socio-linguistically oriented study to explore the 'Arabizi' phenomenon in computer-mediated 
communication among Saudi Arabians. The objective of the study is to understand the following:

1) Why some Saudi Arabians use Arabizi.

2) How Saudi Arabians use Arabizi, specifically with respect to topics, and with whom. And conversely what topics are not discussed with Arabizi.

3) The attitudes of Saudi Arabians towards the use of Arabizi to communicate with one another.

4) The perceived advantages and disadvantages of using Arabizi.

\section{Literature Review}

\subsection{Computer-Mediated Communication (CMC): The Global Context}

Computer-mediated communication (CMC) refers to a form of writing that is primarily technology-based and is predominantly used in the cyberspace. It is defined by Jacob (2008, p. 470) as "the practice of using networked computers and alphabetic text to transmit messages between people or groups of people across space and time". $\mathrm{CMC}$ incorporates a variety of styles of communication the first being synchronous, also known as real-time communication, which is mostly identified when individuals are taking part in instant messaging (MOOs or Internet relay chat) (Herring \& Androutsopoulos, 2015). The second form of writing is asynchronous, also identified as delayed communication, which is experienced via emails or website bulletin boards (Herring \& Androutsopoulos, 2015). This form of conversation has a longer reply period than instant messaging before replies are received; however, depending on the topic of discussion waiting periods with urgent matters highlighting swift acknowledgments. The last form of CMC writing styles is hypermedia, also known as hyper-textual, which is based on linking a variety of people to one conversation (Herring \& Androutsopoulos, 2015). Although CMC is conventionally performed through computers, other technological devices such as mobile phones and tablets are at our disposal for this purpose. Text-based CMC through different devices (e.g., computers, mobile phones) and particularly the sociolinguistic aspects giving rise to different linguistic phenomena in messaging are the focus of this study.

\subsection{The Rise of Messaging Language and (Socio) Linguistic Interest}

In the 1990s, mobile phone manufactures made it possible for a variety of people to own mobile phones beyond the intended market of businesspersons who were in need of a means to communicate while on the move (Marsh, 2005). In Finland, a marketing research study by mobile giant Nokia, which at the time had established itself as a leading mobile device provider, showed that Short Message Service (SMS) was becoming increasingly popular (Marsh, 2005). Evidently, in 1997, the texting bubble developed (Thurlow \& Brown, 2003) and today it has become the most popular communication method around the world with more than 350 billion text messages sent monthly via different mobile applications (Al- Salman, 2017). Texting has even become more popular than voice communication in many places around the world such as Japan and Hong Kong (Thurlow \& Poff, 2011). According to Harmer (2011) for the first time in the history of communication, messages could be sent as well as received with little restrictions of place or time; additionally, this could be done without the knowledge of any nearby party. Grinter and Eldridge (2013) state that this new trend had very little to do with technology, but it is seen as a new form of communication culture that encouraged the expression of thoughts in 160 characters. Research in the past two decades has started studying the text-based CMC in the aim of understanding this emergent and widespread phenomenon, especially among young people and how it affects language (Herring, 2012). Some researchers tried to describe the phenomenon in terms of how similar or different it is from the writing and speaking modalities. For example, Herring (2011, p. 2) maintains that "users experience CMC in fundamentally similar ways to spoken conversation, despite CMC being produced and received by written means". In addition, describing CMC language Kloučková (2013, p. 75) states that it is a "written speech with people writing as if they were speaking". Other scholars have even claimed that the language of the CMC constitutes a third modality, which means that it is totally different from writing and speech. For example, Crystal (2005, p. 1) states that CMC language "differs in fundamental respects from traditional speech and from writing". Because of this, Crystal argues that CMC language should not be considered a mix of writing and speaking, but a third modality which has its own distinct characteristics. This has resulted in a growing body of research attempting to describe how and under what circumstances CMC language is different from writing and speech at both the utterance and discourse levels and cross-linguistically (Herring, 2012).

\subsection{Computer-Mediated Communication (CMC): The Romanization of Arabic}

Arabizi is primarily characterized by the use of Latin script to spell out Arabic words. Why and how Arabizi is used as well as the attitudes towards it and the perceived advantages and disadvantages of its use are the focus of this study and the particular setting of the study is Saudi Arabia. To date, it is believed that only two studies (i.e., 
AbuSa ${ }^{e e} a l e e k, 2014$; Kenali et al., 2016) have been conducted on the use of Arabizi in Saudi Arabia and these are limited in scope (see section 3.5). However, since Arab countries have a shared language and culture, studies on Arabizi in other Arab countries are useful in this context and could enhance our understanding of the phenomenon. This section starts with an overview of two previous attempts to Romanize Arabic and how they were confronted as this might enlighten our exploration of the attitudes towards the use of Arabizi. Following this, previous literature on Arabizi is reviewed. Before the 1990s, there were significant calls for the restructuring of Arabic language particularly in writing through the Romanization of Arabic script with Latin. Throughout the 1880s, Egyptian Linguistic scholars led by Wilhelm Spitta and Karl Vollars argued out a variety of reasons as to the reform of Arabic specifically due to the increasing global use of Latin scripts (Halpern, 2007). However, their preposition was highly criticized and finally rejected due to a variety of reasons. According to Rushdi - Saleh et al. (2011), most of the scholars against the Romanization of Arabic script as considered such notions as unnecessary because of the geographical nature of Arabic nations throughout North Africa and the Gulf and primarily conservation of the Arabic culture (Bassiouney, 2009).

It should be noted that in the 1880 s, the globalization phenomenon was not realized, with most cross-border relation being restrictive to culture and language and then the restructure of Arabic had little significance to the people. Later in the 1990s, Salama Mosa rekindled the discussion of Romanization of Arabic script, his arguments centering on the premise that Latin script was more effective than Arabic script in reference to the academic fields (Mosa, 1998). According to Mosa, the Arabic script is dissociative with globalization as it is one of the very few languages that is related with a religion, Islam; additionally, stating that Arabic was significantly harder to learn for foreign academics (Mosa, 1998). Mosa strongly advocated for a movement that sought to convince native Arabic speakers that the Arabic language was incapable of being used in scientific progress. However, one of his biggest critics was his complete disregard for the noteworthy scientific achievements of Arabic speaking scholars and scientists that had been made during the Islamic Golden Age. Moreover, as argued by Széll (2011), there exists no proof that the Arabic language, particularly its written script, is more challenging to study as a foreign language (AFL) than other languages for instance Chinese. By the same token, there exists no evidence to date that demonstrates the efficiencies of using Latin scripts when transcribing Arabic letters; subsequently, all efforts for Romanization of Arabic were significantly contested by both Muslim enthusiasts as well as Arab nationalists (Muhammed, Farrag, Elshamly, \& Abdel-Ghaffar, 2011). According to Shaalan and Raza (2007), Romanization of Arabic was offensive to the Arabic culture given that as a language the Arabic script was part of ancient civilization lasting over fourteen centuries. However, although the Romanization of Arabic in the 19th and 20th centuries failed, an attempt in the 21st century, i.e., Arabizi, has succeeded.

\subsection{Arabizi: A Linguistic Perspective}

As indicated by Attwa (2012) the term Arabizi is a derivative of the words 'Arabic' and 'Inglizee' or English in Arabic. Yaghan (2008) defines it as a modern-day Arabic 'slang' that switches from English to Arabic conversations. Allehaiby (2013) states that due to its profile Arabizi is predominantly employed in Computer-mediated Communication (CMC), thus defining it as a form of Arabic that uses arithmographemes as an additional feature. Arabizi is considered a system of orthographic representations of the spoken language and this is why there appears to be one orthographic system (shared rules) for Arabizi regardless of in which Arab country it is used, but these representations reflect dialectal differences based on the Arabic dialect the user speaks (Bahrainwala, 2011). Arabizi is described as an encoding system that employs the use of Latin script as well as Arabic numerals in place for Arabic words (Palfreyman \& Khalil, 2013). As presented by Ayish and Mellor (2015), each English letter represents an Arabic phoneme that tends to be similar in pronunciation, on the other hands, the Arabic numerals compensate the Arabic phonemes which do not exist in English; however, they resemble Arabic letters in appearance. In some instances, accent marks are employed to Arabic phenomes along with numerals for instance $b$ is represented by ' 6 '. Figure 1 provides an example communication sample in Arabizi. 


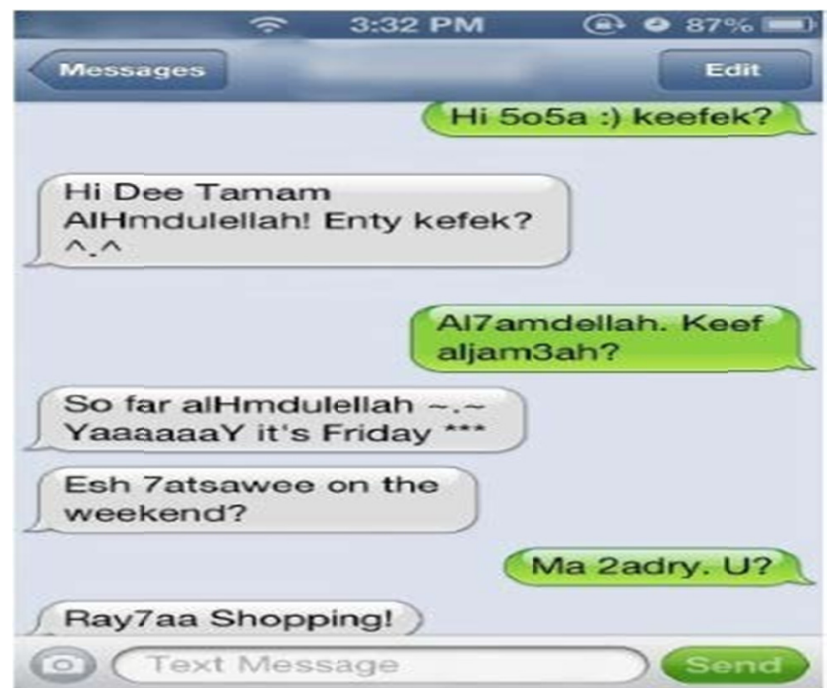

Figure 1. Screenshot of a text conversation that highlights use of Arabizi

Source: Allehaiby, 2013, p. 54.

It is evident in Figure 1. that the two speakers are using both English and Arabic words but in a Latin script. For instance, the third message uses the ' 7 ' instead of the Arabic phoneme $\tau / \hbar /$ to say Alhamdulillah, so it is presented as 'Al7amdellah' ('Thank Allah'). This Arabic letter ( $\tau)$ seems to look similar in appearance to the corresponding number (7); this is also true for most symbols used in Arabizi as the following table of Arabizi symbols shows.

Table 1. Arabizi symbols (source: Allehaiby, 2013, p. 55)

\begin{tabular}{|c|c|c|c|c|}
\hline Phonetic Description & Arabic Letter & Arithmographeme & English Letters & Example Text \\
\hline Voiced glottal stop/?/ & $=$ & 2 & & Bala2 (disaster) \\
\hline $\begin{array}{l}\text { Devoiced pharyngeal } \\
\text { fricative } / \hbar /\end{array}$ & $\tau$ & 7 & $\mathrm{H}$ & 7alwa/halwa (candy) \\
\hline $\begin{array}{l}\text { Voiceless velar } \\
\text { fricative } / \mathrm{x} /\end{array}$ & $\dot{\tau}$ & $5-77$ & $\mathrm{Kh}$ & $\begin{array}{l}\mathrm{Ru} 5 \mathrm{am} / \mathrm{ru} \text { '7am/ } \\
\text { rukham (marble) }\end{array}$ \\
\hline $\begin{array}{l}\text { Voiceless velarized } \\
\text { alveolar fricative/s } \mathbf{s}^{\mathrm{s}}\end{array}$ & ص & 9 & & 9ad (catch) \\
\hline $\begin{array}{l}\text { Voiced velarized dento-alveolar stop } \\
/ \mathrm{d}^{\S} /\end{array}$ & 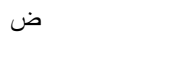 & ‘9 & $\mathrm{D}$ & Ma'9rab/ madrab (racket) \\
\hline $\begin{array}{l}\text { Voiceless velarized dento-alveolarstop } \\
/ \mathrm{t}^{/} /\end{array}$ & b & 6 & & 6alib (student) \\
\hline $\begin{array}{l}\text { Voiced velarized interdental fricative } \\
/ \delta^{\varsigma /}\end{array}$ & b & 6 & & ‘6abi (gazelle) \\
\hline Voiced pharyngeal fricative / / / & $\varepsilon$ & 3 & & La3ib (player) \\
\hline $\begin{array}{l}\text { Voiced uvular } \\
\text { fricative/y/ }\end{array}$ & $\dot{\varepsilon}$ & 3 & & '3areeb (strange) \\
\hline $\begin{array}{l}\text { Voiceless uvular stop } \\
\text { /q/ }\end{array}$ & ق & $\begin{array}{l}8,2 \text { (in some Arabic } \\
\text { dialects) }\end{array}$ & K & $\begin{array}{l}8 \mathrm{arib} / 2 \mathrm{arib} / \\
\text { karib (boat) }\end{array}$ \\
\hline
\end{tabular}

From Table 1 above it is clear that another feature of Arabizi is its extensive use of vowels. According to a study by Attwa (2012), vowels in Arabic are optional and in some instances, they may be omitted altogether in accordance with the speakers' background, context, as well as the number of characters that are supported in one mode of communication to another. For example, Twitter only allows 11 characters subsequently vowels may be omitted or used as presented by the user. Additionally, Altoma (2014) noted that the is a significant dependence in context when it comes to Arabizi considering it is mostly used in informal situations and is airily similar to CMC language which allows the use of shorthand abbreviations such as LOL, ASAP, or YOLO.

\subsection{Previous Studies on Arabizi as a Means of Communication}

There are very few research works which examined code-switching between Arabizi and English (Aboelezz 
2009; Al-Khatib \& Sabbah, 2008; Keong et al., 2015). In Saudi Arabia, there are only two empirical studies which have investigated reasons for Arabizi use (Assalman \& Haraq, 2014; Romaih, 2014). Although these studies were empirical, the results suffered from many limitations. Both studies primarily used a close-ended survey and addressed the general population. The research of Assalman and Haraq (2014) and Romaih (2014), similar to other research reported earlier, warned that Arabizi could negatively affect its users and that institutions in the Saudi society should be aware of these effects in order to minimize the associated risks. Taha (2015) used three focus groups to explore how college students in an Arab country use and perceive Arabizi. This research concludes that the majority of respondents stated that compared to classic Arabic language, Arabizi is more expressive, trendy, and cool. The majority of the respondents also said that lack of adequate Arabic language in private schools, lack of speaking Arabic at home, as well as the smartphones and social media contributed to the wide use of Arabizi. They also stated that the use of Arabizi is a threat to the Arabic language and Arab identity. Most recently, Alghamdi and Petraki (2018) investigated the reasons why young Saudi Arabians use Arabizi online and their attitudes towards its use. This study used 131 questionnaires distributed on social networking sites, and 20 interviews conducted with Saudi users of Arabizi. The findings revealed that the participants use Arabizi because (1), it is the language of their peers, (2) it is cool and stylish, (3) they have difficulties with the Arabic language, and (4) Arabizi constitutes a secret code, allowing escape from judgments of the older generation. The study concludes that Arabizi is a strong marker of Arab youth identity and group solidarity.

\section{Methodology}

\subsection{Participants}

This study used the probability sampling technique to collect data from a sample of 241 participants, who are Saudi Arabians and are aware of and/or use Arabizi in their text-based CMC conversations and thus are considered to be representative of the Arabizi user population. In this study, both sample size and sample scheme were taken into consideration and these were facilitated by the nature of the phenomenon under investigation (Arabizi) and the method used for data collection.

\subsection{Data Collection}

The (online) survey method was used; survey is a very useful technique to collect quantitative data through closed format (multiple choice) and open format questions (Adams \& Cox, 2008). As also pointed out by Adams and Cox, this method allows for obtaining data from a large number of participants particularly when responses are collected through the Internet. Therefore, this technique makes it possible to collect quantitative data and in a relatively short time. This makes it more suitable in the light of the objectives and time limitations of this research. A survey with 38 close-ended and open-ended questions was prepared for this study. The first 9 questions focused on biographical information about gender, age, education, first language background and dialect as well as the knowledge of Arabic and English and writing abilities in both languages. The remaining 29 questions dealt with questions corresponding to the four objectives of the study and in order to draw responses that could answer the research questions. However, the questions in the questionnaire were not organized in the order realized in the objectives but rather in a way to maintain smoothness in moving from one question to another by participants. Eighteen of these questions focused on the participants' knowledge and use of Arabizi with respect to how long and how frequently it is used by them, with whom, how they first heard about it and learned it and what they do when they do not understand part of the message communicated to them in Arabizi (Q: 10-23, 27-30 and 35-36). One question (Q: 24) dealt with the reasons of using Arabizi and this provided multiple choices and the participant was asked to choose as many answers as apply or give additional reasons in a space provided for this purpose. Two open-format questions asked about the topics in which Arabizi is preferred (Q: 25) and not preferred (Q: 26). Further, four closed and open-ended questions focused on participants' attitudes and their perception of the surrounding community attitudes of the use of Arabizi (Q: 3134). Finally, two open format questions explored the perceived advantages (Q: 37) and disadvantages (Q: 38) of the use of Arabizi from the participants' perspectives.

\subsection{Data Analysis}

To analyze this qualitative data, thematic content analysis was conducted. As stated by Maguire and Delahunt (2017), thematic analysis is conducted through finding pattern in the data, identifying themes and organizing data into these themes and then describing, summarizing and reporting them. These steps were followed to analyze the qualitative data in this study. First, responses were obtained from Google forms in an Excel spreadsheet. Responses to the 13 questions were organized in different columns of the spreadsheet and these were dealt with one by one individually. Next, qualitative response columns were scanned to identify answers that were similar 
and patterned together within each column. Following this, these answers which patterned together were given a theme representing them and then columns of responses were checked again to confirm that patterns of answers matched the themes given to them. These themes will be described, summarized and reported in the next section. In reporting direct quotes from our participants, anonymized codes will be used as that AU refers to Arabizi User and ANU refers to Arabizi Non-Users and a number will be added to this. So, for instance, AU10 refers to Arabizi user number 10 and ANU90 is Arabizi non-user number 90.

\section{Results}

\subsection{Reasons for Using or Rejecting Arabizi}

This section reports the reasons our participants stated for embracing Arabizi as a communication system in their text-based CMC conversations. It also presents the reasons given by the non-users who were excluded from the study for their rejection of this system. Nine different reasons were revealed by the study for the use of Arabizi, which are summarized in Figure 2 below. The figure shows that more than a half of the participants $(\mathrm{N}=39 ; 54.9 \%)$ thought that they communicated in Arabizi because it is a communication code among their generation and this was the most widely pointed out reason by our participants. Another relatively popular reason revealed by the results was the lack of Arabic keyboard; this was referred to by about one third $(\mathrm{N}=23 ; 32.4 \%)$ of the respondents. In addition, some participants $(\mathrm{N}=10 ; 14.1 \%)$ believed that they felt they could freely express themselves better in Arabizi more than in Arabic. A slightly lower number of respondents $(\mathrm{N}=8 ; 11.3 \%)$ thought that replicating the western culture was their reason for using Arabizi. Moreover, a reason indicated by $6(8.5 \%)$ respondents was that the Latin script is easier to use than the Arabic one. Other reasons that were mentioned by a smaller number of participants were to communicate with those who speak but cannot read Arabic (5 participants; $7 \%$ ), for fun ( 2 participants; $2.8 \%$ ), to do what others do (2 participants; $2.8 \%$ ) and because it is a new experience (1 participant; $1.4 \%)$.

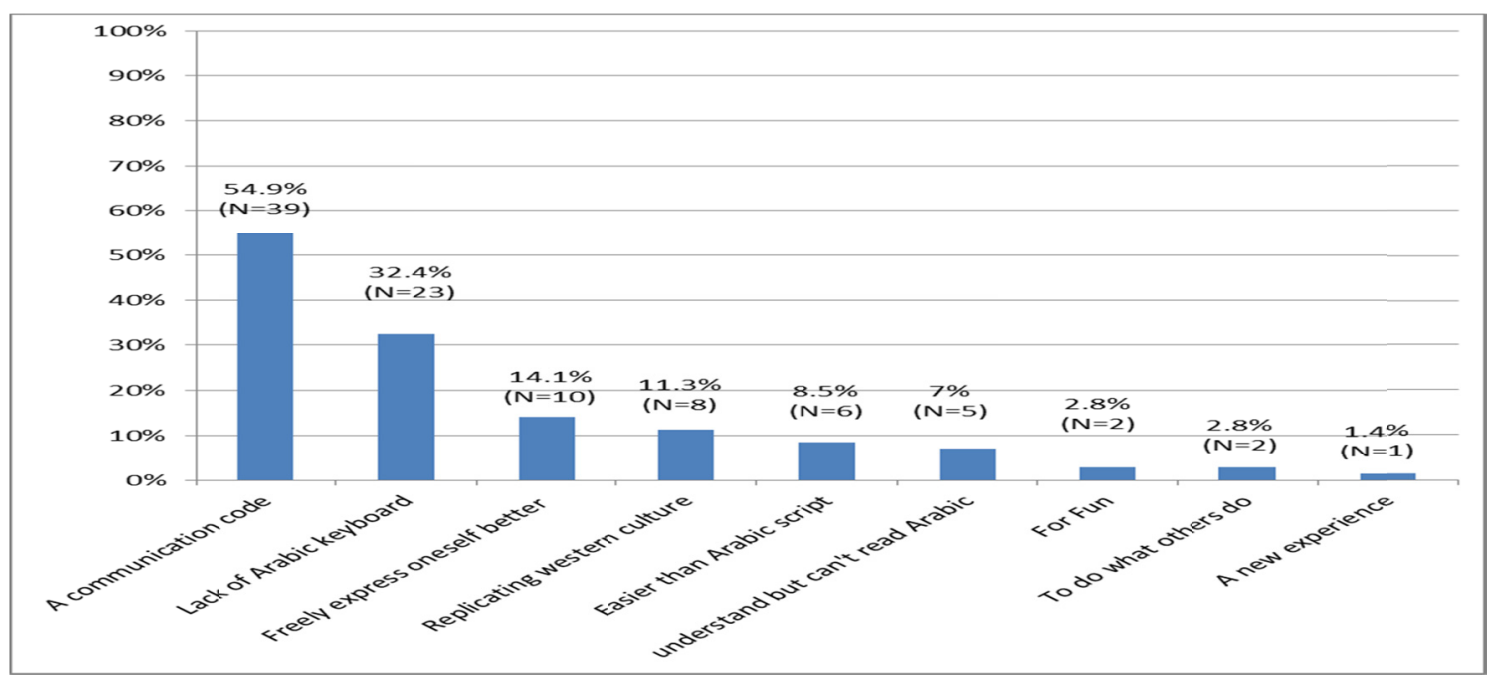

Figure 2. Reasons for using or rejecting Arabizi

On the other hand, the participants $(\mathrm{N}=161)$ who did not use Arabizi mentioned a number of reasons for their rejection of this communication system. These reasons were grouped under seven general themes as follows:

1) They were proud of Arabic and therefore preferred to use it in their CMC communication (e.g., "I like Arabic language" (ANU10); "I am proud of Arabic language" (ANU121)).

2) They thought Arabizi was not suitable to be used (e.g., "It is not suitable" (ANU7); "It is not suitable for my age" (ANU14); "It is the wrong way to express Arabic" (ANU57)).

3) They did not feel the need to use Arabizi ("I do not have a practical reason to use it" (ANU5); "There is no need for it" (ANU61); "Not all people understand it" (ANU2); "not popular among people" (ANU8); "I am not convinced to use it" (ANU48); "all people I contact understand Arabic" (ANU154)).

4) They could express themselves better in Arabic or English (e.g., "The Arabic language is clearer and can express meanings better" (ANU16); "I can express myself in both Arabic and English. using Arabizi might not 
give the meaning I want" (ANU25).

5) They believed that Arabizi was difficult to use (e.g., "It is difficult to use" (ANU62); "It is difficult to read" (ANU86); "If the other person does not understand it, it takes longer to convey what I want" (ANU150).

6) They thought that the use of Arabizi had a negative damaging impact on the Arabic language (e.g., "it is damaging Arabic language" (ANU11); "it will put an end to the use of Arabic language" (ANU20); "it is a danger for Arabic language" (ANU91); "it is a threat to Arabic language and identity" (ANU99); "it is damaging Arabic culture" (153); "it has a negative influence on spelling" (ANU134); "to maintain the purity of Arabic language" (ANU58).

7) They believed that Arabizi was an insult to the other user or the Arabic language (e.g., "it is an insult to the user" (ANU23); "it is an insult to Arabic language" (ANU19); "it is an insult to the language of Quran" (ANU44).

\subsection{The Social Context of Arabizi}

This section reports the results about how Arabizi is used with regard to its social context. It will particularly focus on with whom participants used it and the topics of their communications and conversely with whom they would not use it and what topics they would avert to talk about. The survey results showed that friends and strangers in online platforms were the first points of contact in Arabizi for a large number of participants in the study. Figure 3 below shows that $84.5 \%$ of the participants indicated that they used Arabizi with their friends first and $41.7 \%$ of them also mentioned that they did so with strangers through chatting online. By contrast, only 8.5\% said their siblings were the first to contact using Arabizi and still none chose parents. Indeed, in a different occasion, all participants, except one, said their parents did not use Arabizi, whereas more than three quarters (77.8\%) of the participants confirmed that their friends used Arabizi.

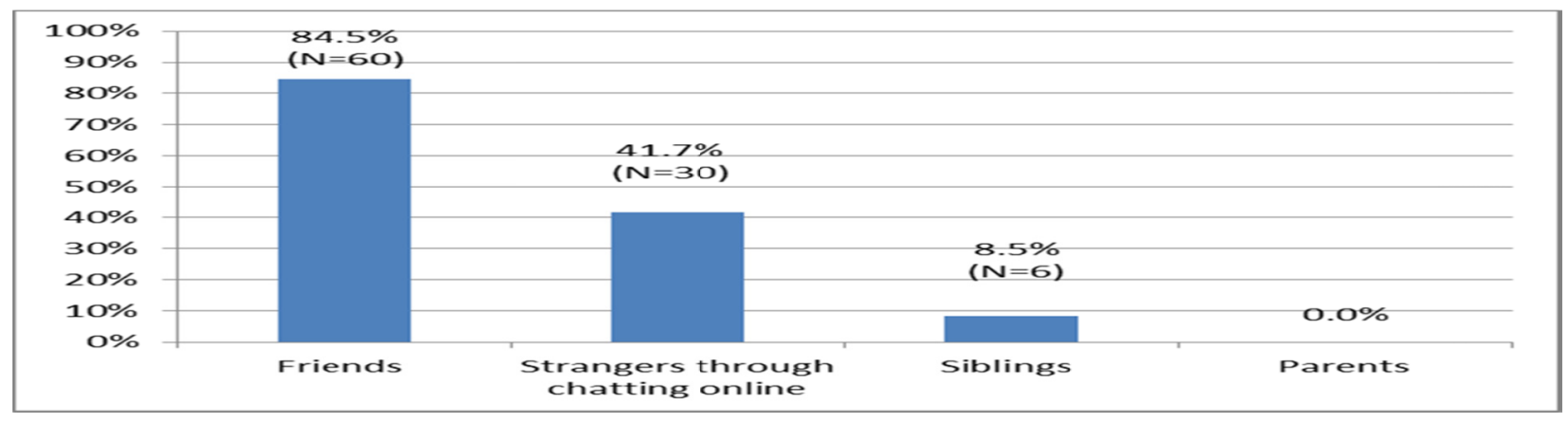

Figure 3. The Social Context of Arabizi

In another open-ended question asking participants to list all people they communicated with using Arabizi and which was responded to by 58 participants, the answers fell in four categories; these are 1) Friends, 2) strangers over the internet, 3) Siblings and other relatives of the same age and 4) People who understand but cannot read Arabic. One participant (i.e., AU72) explained what she meant by 'people who understand but can't read Arabic' as foreigners who have been working in Saudi Arabia for a long time. By contrast, in a different question asking about with whom participants had never used Arabizi, many respondents referred to parents and old people. Some of these respondents mentioned the reason for this as that these people "would not understand Arabizi" (AU2). Other participants also stated that they had not used Arabizi with colleagues at work and teachers and lecturers at university because "it is not appropriate to use Arabizi in formal communications" (AU10). Moving to the topics of communications in which Arabizi is used, these fell into three categories as follows: 1) Occasional conversations among friends (e.g., "greetings" (AU4); "to arrange for a meeting with friends" (AU5); "for socialization and daily topics"; "social topics" (AU12)); 2) private topics among friends (e.g., "when I want to discuss a problem freely with a friend" (AU45); "private topics that I don't want my family to know about" (AU21); "secrets I share with my friends" (AU63); "dating" (AU72); "to express my feelings" (AU11)); 3) general topics in public discussions and platforms (e.g., "to discuss a new event" (AU24); "fashion" (AU3); "sport" (AU36); "topics in gaming chat platforms" (AU16)). On the other hand, a number of topics were mentioned by the participants as not to be written in Arabizi. Some participants stated "serious matters" (AU1) or "important matters" (AU6). 


\subsection{Arabizi Users' and Non-Users' Attitudes}

This section reports the results about the attitudes expressed by participants about Arabizi use based on their response to two questions in the survey. Results arising from a fourth question dealing with their perception of older people's attitude toward this phenomenon are also presented here. In one question which asked participants to indicate whether Arabizi was a bad or good phenomenon and to provide a reason for their answers, 55 participants responded. Twenty-six (47.3\%) of these thought it was good and the reasons provided were in line with the following:

"It facilitates learning English and English letters" (AU36).

"It gives freedom in self-expression" (AU12).

"It allows for expressing feelings freely without the fear of detection when messaging the other gender" (AU53).

"It is easy" (AU9).

"It allows for understanding other Arabic dialects" (AU72).

By contrast, 17 respondents (30.9\%) had the attitude that Arabizi was bad for reasons that were consistent with the following:

"It is a sign of abandoning mother tongue" (AU1).

"It leads to changing Arabic language" (AU4).

"It destroys the ability in using Arabic" (AU17).

"It leads to the loss of Arab identity and culture" (AU14).

"It is difficult to read and write in Arabizi" (AU32).

"Arabic loses its purity" (AU45).

In addition, a great majority of the 161 non-users expressed attitudes in line with these negative attitudes of users. However, these non-users wrote a harsher language to express their attitudes. Some of them said the following: "it is superficial" (ANU103), "silly phenomenon" (ANU2), "it indicates ignorance" (ANU4), "it is disgusting" (ANU112) and "it is a stupid language and used solely by teens" (ANU152). However, 12 participants (21.8\%) expressed their attitudes in neutral terms. They provided statements such as "it is neither good nor bad" (AU18), "every one expresses meaning in the way s/he likes" (AU21), "it is a personal preference" (AU12) and "it is just fun" (AU60). Furthermore, approximately equal numbers of participants believed that Arabizi is a cultural phenomenon that would endure for many years (23 out of 47 participants; $49 \%$ ) or would end soon (24 out of 47 participants; $51 \%$ ). Those who believed that it would endure held this belief because i) it is easier, ii) it is a new version of written Arabic iii) there is a mix between and iv) it is very popular among the new generation, who decides what happens in the future. On the other hand, the respondents who thought it would end soon attributed this to reasons such as i) writing in Arabic has become possible in software programmes and social media platforms, ii) its use is restricted to teens and when they get older, they will abandon it, iii) it is just trendy now and will end soon and iv) teaching English has improved and people will start to use either Arabic or English. The great majority of our participants $(80 \%)$ perceived the older generation to hold negative attitudes toward Arabizi. The possible reasons for such attitudes were thought to be as follows:

1) They fear the loss of Arab identity and culture

2) They consider it a sign of abandoning identity and culture

3) They consider it a blind imitation of the western culture

4) It is an insult to Arabic language

In contrast, only $12 \%$ of the participants perceived the older generation to hold positive attitudes, with the rest $8 \%$ being unsure about this. The following section presents the results about the participants' perceptions about the advantages and disadvantages of Arabizi.

\subsection{The Perceived Advantages and Disadvantages of Arabizi Use}

One close-ended question listing some advantages and disadvantages that there were revealed by previous studies was included in the questionnaire. However, to allow participants to provide their own responses, two open-ended questions focusing on each of these aspects separately were also included. Starting with the results of the close-ended question, it was found that more participants held negative beliefs with regard to Arabizi use. Forty-six out of 65 participants $(70.8 \%)$ believed that youngsters risk losing their writing ability in Arabic 
language when they use Arabizi (43.1\%) or that the Arabic language is merging with English, and it's losing its identity (27.7\%). By contrast, only 19 participants (29.2\%) embraced the positive belief that Arabizi makes communication easy and modernizes their language. However, the open-ended questions revealed a wider range of advantages and disadvantages. As for the advantages, participants' responses were consistent with what was found in questions probing their reasons for using Arabizi or reasons for their positive attitudes toward the phenomenon. These were as follows:

It allows "easier communication among the new generation" (AU3)

It is "faster in writing" (AU31) and "better than switching forth and back between Arabic and English keyboards" (AU21)

"It is fun" (AU60)

It is "a communication code among friends and brothers" (AU6) and "it is difficult to read by older people, so parents cannot read messages" (AU59) and therefore "it allows teens to express what they want" (AU27)

"It facilitates communication with those who understand but cannot read Arabic" (AU10)

"It helps those who don't have an Arabic keyboard" (AU7)

On the other hand, some disadvantages were also uncovered. These are provided below:

"It leads to the loss of Arab identity" (AU3)

"It is a threat to Arabic language" (AU14)

"It leads to spelling problems when writing in Arabic or English" (AU5)

"It leads to lack of ability to express oneself in Arabic" (AU8)

"It does not have a standard form and anyone can invent new forms" (AU46)

"It lacks eloquence" (AU1)

"It is not understood by all" (AU21)

It is important to note that these disadvantages were also similar to those mentioned by the 161 nonusers who responded the questionnaire.

\section{Discussion}

The finding reported revealed that Arabizi was used by our participants for a set of different reasons although some reasons were more popular than others. The first and most popular reason indicated by more than half of the participants was that Arabizi was considered a communication code among their generation. This might mean that they use it because it ensures their privacy and helps them perform a social identity. This reason was not revealed by any of the previous Arabizi studies apart from Palfreyman and Al-Khalil (2003), who pointed out that some of their Emirati participants, stated that the use of privacy could ensure their privacy. This is also consistent with what was proposed for the use of textisms in English. For example, Wray (2002) maintained that performing social identity is an important reason of the use of textisms. The lack of the Arabic keyboard was the second most popular reason for Arabizi use by our participants. This confirms what was found by Yaghan (2008), who revealed that the absence of Arabic keyboard was the main reason for his Jordanian university students to use Arabizi. However, none of the other Arabizi studies in other Arab countries found this. A third reason mentioned by some of our participants was that Arabizi was more expressive than Arabic. This refers to users' ability to express their dialects in a written form and their feelings. Indeed, the same finding was reached by Yaghan (2008) in Jordan, and Bahrainwala (2011) and Taha (2015) in the United Arab Emirates. This is also consistent with the proposal of Androutsopoulos (2011) that in CMC communications, users resort to textisms, such as the use of the phonetic rather than orthographic spelling of a word, to sound as if they were speaking, and thus, informal. According to Tagg (2009), informality in communication might be performed because users tend to be intimate in their text-based communications.

Concerning the social context of Arabizi, it seems to be widespread among educated people who range in age between 18 and 35 years. Indeed, the results showed that the majority of those who used Arabizi were between 18 and 35 years old $(86.1 \%)$ and only a minority (13.9\%) was over 35 years old. In addition, $87.5 \%$ of the participants were graduate or postgraduate students or degree holders. Although this might reflect the age range and educational degree level of the participants of the study rather than the actual age range and educational degree level of the people who use Arabizi, this seems to be consistent with Abu Sa"aleek's (2014) study, which found that Arabizi was widespread among university students. Also, this is consistent with what Palfreyman and 
Al- Khalil's (2003) participants reported that Arabizi would be used mainly among educated people. However, further research, particularly qualitative and large scale, is needed to confirm this finding. Moreover, Arabizi seems to be the communication system primarily used among friends and individuals of the same age and mainly in informal contexts. This appears from the finding that social media and friends were the two main sources from which participants heard about and learned Arabizi. In addition, the two widely cited points of contacts that participants used Arabizi with were friends and strangers in social media platforms; only $8.5 \%$ of participants mentioned siblings of the same age as points of contact in Arabizi and none mentioned using it with parents, older people, colleagues at work, teachers at school or university. Support for this also comes from the results of another question in which participants stated that they used Arabizi neither with parents or older people because these would not understand Arabizi nor with colleagues at work because Arabizi was thought not to be appropriate for formal contexts. This is compatible with the findings of Bahrainwala (2011), whose Emirati participants stated that they would not use Arabizi in formal relations.

With regards to attitudes toward Arabizi, the results revealed that a third of participants thought it was a bad phenomenon and still about $20 \%$ of them were neutral. Those who perceived Arabizi positively held beliefs about its power to facilitate communication, to give freedom of self-expression and allow for understanding other Arabic dialects. On the other hand, negative attitude holders focused in their expressed beliefs about Arabizi damaging effects on Arabic culture and language. In addition, neutral attitude holders stated that it is neither good nor bad as it is a personal preference which communication system to use. This seems to be partly consistent with some previous literature as while in some studies (Al-Haq, 2015; Palfreyman \& Al-Khalil, 2003) only positive attitudes prevailed among participants, in other studies (Bahrainwala, 2011) only negative attitudes were expressed by participants. Yet, Taha's (2015) study participants held both positive and negative attitudes at the same time, which was closer to what was found in our study. While it is not clear why some studies found mainly positive or mainly negative attitudes, what seems to be important is that both attitudes exist, and our study provides evidence for this. However, what needs interpretation is why some users communicate in Arabizi although they see it as a negative phenomenon. Two possible interpretations can be provided here. First, it might be the case that they really did not want to use Arabizi because they viewed it negatively as damaging to their mother tongue, but they used it because they found themselves forced to communicate thorough it as their friends used it when they contacted them or they did not have Arabic keyboards in the communication devices they owned. What validates this interpretation is that most participants mentioned that their friends used Arabizi when they contacted them and about a third of the participants indicated that the absence of Arabic keyboard was their reasons for using Arabizi.

The perceived advantages and disadvantages of Arabizi use revealed to be widely consistent with participants' reasons for using Arabizi and the beliefs they used to justify their positive attitudes. Conversely, the disadvantages were also compatible with participants' reasons for rejecting to use Arabizi and the beliefs they used to justify their negative attitudes. One of the advantages of Arabizi was that it is easier and faster to use in communication between people. As mentioned above, this was mentioned by some participants as their reason for using Arabizi, but it was not as popular as other reasons. Another important and more popular advantage was that Arabizi is a communication code among the young generation and it cannot be understood by the older generation. Indeed, the privacy issue seemed to be very important to these participants as some stated "it is difficult to read by older people, so parents cannot read messages" (AU59) and "it allows teens to express what they want" (AU27). It was argued in section 5.2 above that ensuring privacy and performing a social identity could be one of the most important triggers for the rise of Arabizi or adopting it by youths. This seems to be supported further by this advantage mentioned by our participants. Still for others, the advantage of Arabizi was that it compensates for the absence of the Arabic keyboard from technological devices. By contrast, most of the disadvantages mentioned by participants were relating to its damaging effect on the Arabic language and culture and on users' linguistic abilities in spelling and self-expression in Arabic. These disadvantages were held by some of those who used Arabizi. Yet, these were the same disadvantages mentioned by those who refused to use Arabizi. So, these disadvantages seemed to be effective on some participants to the extent that they averted from using Arabizi.

\section{Conclusion}

The present study aimed to explore some sociolinguistic aspects of a phenomenon called Arabizi, which is a linguistic system used by Saudi youths in their CMC communications. The literature review showed that studies conducted in the Saudi context focused primarily on the linguistic aspects of the phenomenon and studies in other Arab contexts such as Jordan and United Arab Emirates were found to be limited in scope necessitating and not providing sufficient information about the phenomenon. To bridge the gap in previous research, a study 
was designed to explore different sociolinguistic aspects of the phenomenon related to why it is used, how it is used with respect to with whom it is used and the topics in which it is used or not used, the attitudes of its users toward its use and the perceived advantages and disadvantages of its use. A survey was developed and posted online in public Saudi twitter webpages and through WhatsApp to groups of friends. This research is believed to have made an original contribution to knowledge about the linguistic phenomenon under study: Arabizi. First, this is the first sociolinguistic study on Arabizi in Saudi Arabia. Second, different from previous studies on the use of Arabizi in other Arab countries. This study is wider in scope as it explored a number of sociolinguistic aspects (i.e., why and how Arabizi is used, the attitudes of its users toward its use and their perceived advantages and disadvantages of its use) that were not looked into in one single study. Exploring all of these aspects together in this study provides betters insights into this phenomenon.

\section{Acknowledgements}

I am sincerely and heartily grateful to my supervisor, Dr. Christopher Shank, for his continuous encouragement and supportive guidance throughout my research period.

\section{References}

Aboelezz, M. (2012). We are young. We are trendy. Buy our product! The use of Latinized Arabic in edited printed press in Egypt. United Academics Journal of Social Sciences, 2, 48-72. Retrieved from http://www.lancs.ac.uk/pg/aboelezz/docs/WeAreYoungTrendy.pdf

Aboelezz, M. (2015). We are young. We are trendy. Buy our product! Retrieved October, 11.

AbuSa ${ }^{e e}$ aleek, A. (2014). Latinization of arabic language in the electronic communication: concept and practice. Aligarh Journal of Linguistics, 4, 75-89.

Adams, A., \& Cox, A. L. (2008). Questionnaires, in-depth interviews and focus groups.

Allehaiby, W. H. (2013). Arabizi: An Analysis of the Romanization of the Arabic Script from a Sociolinguistic Perspective. Arab World English Journal, 4(3).

Al-Salman, S. M., \& Saeed, A. T. (2017). Effects of text-messaging on the academic writing of Arab EFL students. Research in Language, 15(3), 237-252. https://doi.org/10.1515/rela-2017-0014

Alshenqeeti, H. (2014). Interviewing as a data collection method: A critical review. English Linguistics Research, 3(1), 39-45. https://doi.org/10.5430/elr.v3n1p39

Altoma, S. J. (1974). Language education in Arab countries and the role of the academies. Advances in Language Planning, 279-313. https://doi.org/10.1515/9783111583600.279

Androutsopoulos, J. (2011). Language change and digital media: a review of conceptions and evidence. Standard languages and language standards in a changing Europe, 145-161.

Attwa, M. (2012). Arabizi: A writing variety worth learning. The American University in Cairo, School of Humanities and Social Science.

Ayish, M., \& Mellor, N. (2015). Reporting in the MENA region: cyber engagement and pan-Arab social media. Rowman \& Littlefield.

Bahrainwala, L. (2011). You Say Hello, I Say Mar7aba: Exploring the Digi-speak that Powered the Arab Revolution. Michigan State University. Rhetoric and Writing.

Baron, N. S. (2010). Discourse structures in instant messaging: The case of utterance breaks. Language@ Internet, 7(4).

Bassiouney, R. (2009). Arabic sociolinguistics. Edinburgh University Press. https://doi.org/10.3366/edinburgh/9780748623730.001.0001

Bianchi, R. M. (2012). 3arabizi-When local Arabic meets global English. Acta Linguistica Asiatica, 2(1), 89-100. https://doi.org/10.4312/ala.2.1.89-100

Bies, A., Song, Z., Maamouri, M., Grimes, S., Lee, H., Wright, J., ... \& Rambow, O. (2014, October). Transliteration of arabizi into arabic orthography: Developing a parallel annotated arabizi-arabic script sms/chat corpus (pp. 93-103). In Proceedings of the EMNLP 2014 Workshop on Arabic Natural Language Processing (ANLP). https://doi.org/10.3115/v1/W14-3612

Bieswanger, M. (2015). 2 abbrevi8 or not 2 abbrevi8: A contrastive analysis of different shortening strategies in English and German text messages. SALSA XIV. 
Chinnery, G. M. (2006). Emerging technologies: Going to the MALL: Mobile assisted language learning. Language Learning \& Technology, 10(1), 9-16.

Creswell, J. W., \& Creswell, J. D. (2017). Research design: Qualitative, quantitative, and mixed methods approaches. Sage publications.

Crystal, D. (2005, February). The scope of Internet linguistics (pp. 17-21). In Proceedings of American Association for the Advancement of Science Conference; American Association for the Advancement of Science Conference, Washington, DC, USA.

Crystal, D. (2008). Texting. ELT Journal, 62(1), 77-83. https://doi.org/10.1093/elt/ccm080

Cvjetkovic, S. (2010). Computer-Mediated Communication: A Study of Language Variation on Internet Chat. rapport nr.: SPL kandidatuppsats i engelska SPL 2010-005.

Danesi, M. (2016). The semiotics of emoji: The rise of visual language in the age of the internet. Bloomsbury Publishing.

Darwish, K. (2013). Arabizi detection and conversion to Arabic (pp. 217-224). Proceedings of the EMNLP 2014 Workshop on Arabic Natural Language Processing (ANLP). https://doi.org/10.3115/v1/W14-3629

Derks, D., Bos, A. E., \& Von Grumbkow, J. (2008). Emoticons in computer-mediated communication: Social motives and social context. Cyber Psychology \& Behavior, 11(1), 99-101. https://doi.org/10.1089/cpb.2007.9926

Dresner, E., \& Herring, S. C. (2010). Functions of the nonverbal in CMC: Emoticons and illocutionary force. Communication Theory, 20(3), 249-268. https://doi.org/10.1111/j.1468-2885.2010.01362.x

Farina, F., \& Lyddy, F. (2011). The language of text messaging: "Linguistic ruin" or resource? Irish Psychologist, $37(6), 145-149$.

Fricker, R. D. (2017). Sampling Methods for Online Surveys. In N. Fielding, R. M. Lee \& G. Blank (Eds.), The SAGE Handbook of Online Research Methods (2nd ed.). London: Sage.

Ghanem, R. (2011). Arabizi is destroying the Arabic language. Arab News, 20.

Grinter, R., \& Eldridge, M. (2003, April). Wan2tlk? everyday text messaging (pp. 441-448). In Proceedings of the SIGCHI conference on Human factors in computing systems. ACM. https://doi.org/10.1145/642611.642688

Guetterman, T. (2015). Descriptions of sampling practices within five approaches to qualitative research in education and the health sciences.

Halpern, J. (2007). The challenges and pitfalls of Arabic romanization and Arabization. In Proc. Workshop on Comp. Approaches to Arabic Script-based Lang.

Harmer, J. (2011). The practice of English language teaching. Harlow: Pearson Longman.

Herring, S. C. (2011). Computer-mediated conversation Part II: Introduction and overview.Language@ Internet, $8(2)$.

Herring, S. C. (2012). Grammar and electronic communication. The Encyclopedia of Applied Linguistics, 1-9. https://doi.org/10.1002/9781405198431.wbeal0466

Herring, S. C. (2013). Discourse in Web 2.0: Familiar, reconfigured, and emergent. Discourse, 2, 1-25.

Herring, S. C., \& Androutsopoulos, J. (2015). Computer-mediated discourse 2.0. The Handbook of Discourse Analysis, 2, 127-151. https://doi.org/10.1002/9781118584194.ch6

Jacobs, G. E. (2008). People, purposes, and practices: Insights from cross-disciplinary research into instant messaging. The Handbook of Research on New Literacies, 467-490.

Jaran, S. A., \& Al-Haq, F. A. A. (2015). The Use of Hybrid Terms and Expressions in Colloquial Arabic among Jordanian College Students: A Sociolinguistic Study. English Language Teaching, 8(12), 86-97. https://doi.org/10.5539/elt.v8n12p86

Kahari, L. (2014). Language of texting, patterns and factors of language choice in text messaging of University of Zimbabwe, Shona-English bilinguals. Language, 3.

Kemp, N., \& Bushnell, C. (2011). Children's text messaging: Abbreviations, input methods and links with literacy. Journal of Computer Assisted Learning, 27(1), 18-27. https://doi.org/10.1111/j.1365-2729.2010.00400.x 
Kenali, A. M. S., Yusoff, N. M. R. N., Kenali, H. M. S., \& Kamarudin, M. Y. (2016). Code-Mixing Consumptions among Arab Students. Creative Education, 7(7), 931. https://doi.org/10.4236/ce.2016.77097

Kent, S., \& Johnson, G. (2012). Differences in the linguistic features of text messages send with an alphanumeric multi-press keypad mobile phone versus a full keypad touchscreen smartphone. Scottish Journal of Arts, Social Sciences and Scientific Studies, 7(1), 50-67.

Khedher, M. Z., Abandah, G. A., Al-Anati, W. A., Ababneh, S. M., Zghoul, A. A., \& Hattab, M. S. (2015, November). Effect of topic on the Arabic language used on social networks and mobile phone communications (pp. 1-6). In 2015 IEEE Jordan Conference on Applied Electrical Engineering and Computing Technologies (AEECT). IEEE. https://doi.org/10.1109/AEECT.2015.7360593

Kloučková, V. (2013). The degree of spookiness in two modes of CMC. Theory \& Practice in English Studies, $6(2), 75-92$.

Lanchantin, T., Simoës-Perlant, A., \& Largy, P. (2014). Good Spellers Write more Textism than Bad Spellers in Instant Messaging: The Case of French. Psych Nology Journal, 12.

Maguire, M., \& Delahunt, B. (2017). Doing a thematic analysis: A practical, step-by-step guide for learning and teaching scholars. AISHE-J: The All Ireland Journal of Teaching and Learning in Higher Education, 9(3).

Marsh, J. (Ed.). (2005). Popular culture, new media and digital literacy in early childhood. Psychology Press. https://doi.org/10.4324/9780203420324

Maxwell, J. A. (2013). Research design: An interactive approach. Social Sciences, 14(7), 36-42.

McKim, C. A. (2017). The value of mixed methods research: A mixed methods study. Journal of Mixed Methods Research, 11(2), 202-222. https://doi.org/10.1177/1558689815607096

Miličević, M., Ljubešić, N., \& Fišer, D. (2017). Birds of a feather don’t quite tweet together: An analysis of spelling variation in Slovene, Croatian and Serbian twitterese. Investigating computer-mediated communication: Corpus-based approaches to language in the digital world, 14-43.

Mosa S. (1998). Translating names and technical terms in Arabic text (pp. 34-41). In Proceedings of the Workshop on Computational Approaches to Semitic Languages. Association for Computational Linguistics.

Muhammed, R., Farrag, M., Elshamly, N., \& Abdel-Ghaffar, N. (2011). Summary of Arabizi or Romanization: The dilemma of writing Arabic texts. Jīl Jadīd Conference, Austin, TX. Retrieved from http://www.utexas.edu/cola/depts/mes/events/conferences/jiljadid2011/papers/FinalArabizSummary_JilJadi d.pdf

Nowell, L., Norris, J., White, D., \& Moules, N. (2017). Thematic Analysis. International Journal of Qualitative Methods, 16(1), 1-13. https://doi.org/10.1177/1609406917733847

Onwuegbuzie, A. J., \& Collins, K. M. (2007). A typology of mixed methods sampling designs in social science research. The Qualitative Report, 12(2), 281-316.

Opdenakker, R. (2006, September). Advantages and disadvantages of four interview techniques in qualitative research (Vol. 7, No. 4). In Forum Qualitative Sozialforschung/Forum: Qualitative Social Research.

Palfreyman, D., \& Khalil, M. A. (2013). “A Funky Language for Teenzz to Use:” Representing Gulf Arabic in Instant Messaging. Journal of Computer-Mediated Communication, 9(1), JCMC917. https://doi.org/10.1111/j.1083-6101.2003.tb00355.x

Plester, B., Wood, C., \& Joshi, P. (2009). Exploring the relationship between children's knowledge of text message abbreviations and school literacy outcomes. British journal of Developmental Psychology, 27(1), 145-161. https://doi.org/10.1348/026151008X320507

Rushdi - Saleh, M., Martín - Valdivia, M. T., Ureña - López, L. A., \& Perea - Ortega, J. M., (2011). OCA: Opinion corpus for Arabic. Journal of the Association for Information Science and Technology, 62(10), 2045-2054. https://doi.org/10.1002/asi.21598

Sampson, D. G., Isaias, P., Ifenthaler, D., \& Spector, J. M. (Eds.). (2012). Ubiquitous and mobile learning in the digital age. Springer Science \& Business Media. https://doi.org/10.1007/978-1-4614-3329-3

Savin-Baden, M., Claire, H., \& Major, C. (2013). Qualitative Research: The Essential Guide to Theory and Practice.

Schoonenboom, J., \& Johnson, R. B. (2017). How to construct a mixed methods research design. KZfSS Kölner $\begin{array}{lllll}\text { Zeitschrift für Soziologie und } & \text { Sozialpsychologie, }\end{array}$ 
https://doi.org/10.1007/s11577-017-0454-1

Shaalan, K., \& Raza, H. (2007). Person name entity recognition for Arabic (pp. 17-24). In Proceedings of the 2007 Workshop on Computational Approaches to Semitic Languages: Common Issues and Resources. Association for Computational Linguistics. https://doi.org/10.3115/1654576.1654581

Spilioti, T. (2009). Graphemic representation of text-messaging. Pragmatics. Quarterly Publication of the International Pragmatics Association (IPrA), 19(3), 393-412. https://doi.org/10.1075/prag.19.3.05spi

Széll, M. (2011). Westernizing Arabic: Attempts to "simplify" the Arabic script. Tipográfiai Diákkonferencia, 2012.

Tagg, C. (2009). A corpus linguistics study of SMS text messaging. Doctoral dissertation, University of Birmingham.

Taha, M. (2016). Arabizi: Is Code-Switching a Threat to the Arabic Language. Proceeding of the Asian Conference on Arts \& Humanities. Retrieved July 20, 2018, from http://papers.iafor.org/wp-content/uploads/papers/acah2015/ACAH2015_13058.pdf

Thurlow, C., \& Brown, A. (2003). Generation Txt? The sociolinguistics of young people's text-messaging. Discourse Analysis Online, 1(1), 30.

Thurlow, C., \& Poff, M. (2011). The Language of Text-Messaging. In S. Herring, S. Dieter \& T. Virtanen (Eds.), Handbook of the Pragmatics of CMC. Berlin and New York: Mouton de Gruyter.

Trotta, J., \& Danielson, M. (2011). Image-based online communication observations on the status of images as linguistic constituents in computer mediated communication. In conference proceedings McLuhan Galaxy Conference: Understanding Media, Today. Barcelona, May 23rd-25th.

Verheijen, L. (2017). WhatsApp with social media slang? Youth language use in Dutch written computer-mediated communication (pp. 72-101). In Investigating Computer-Mediated Communication: Corpus-Based Approaches to Language in the Digital World. Ljubljana University Press.

Walliman, N. (2011). Research theory, Research methods: the basics. https://doi.org/10.4324/9780203836071

Yaghan, M. A. (2008). "Arabizi”: A contemporary style of Arabic Slang. Design Issues, 24(2), 39-52. https://doi.org/10.1162/desi.2008.24.2.39

Zohrabi, M. (2013). Mixed Method Research: Instruments, Validity, Reliability and Reporting Findings. Theory \& Practice in Language Studies, 3(2). https://doi.org/10.4304/tpls.3.2.254-262

\section{Copyrights}

Copyright for this article is retained by the author, with first publication rights granted to the journal.

This is an open-access article distributed under the terms and conditions of the Creative Commons Attribution license (http://creativecommons.org/licenses/by/4.0/). 